\title{
La coronarioectasia difiere en los factores de riesgo clásicos de enfermedad coronaria
}

\author{
Alberto Fuensalida ${ }^{1,4}$, Dante S Lindefjeld ${ }^{1,2,3}$, Arturo Giacaman ${ }^{1,4}$ René Hameau ${ }^{1,4}$, Martín Valdebeni- \\ to ${ }^{1,5}$, Manuel Méndez ${ }^{1,2,3}$, Nicolás Veas ${ }^{3}$, José Luis Winter ${ }^{3}$, Gonzalo Martínez ${ }^{1,2,3}$, Osvaldo Pérez ${ }^{1,2,3}$, \\ Alejandro Martínez ${ }^{1,2}$. \\ 1. Departamento de Enfermedades Cardiovasculares, Pontificia Universidad Católica de Chile. \\ 2. Centro de Terapia Endovascular, Hospital Clínico de la Universidad Católica de Chile. \\ 3. Laboratorio de Hemodinamia Hospital Sótero del Río. \\ 4. Residente de Cardiología, Pontificia Universidad Católica de Chile. \\ 5. Residente de Cardiología Intervencional, Pontificia Universidad Católica de Chile.
}

Introducción: La coronarioectasia (CE), es una infrecuente forma de enfermedad coronaria, en que dilataciones coronarias coexisten con placas ateroescleróticas. Puede presentarse como cuadros agudos o crónicos, aún sin estenosis significativa. Distintas series lo han asociado a perfiles variados de factores de riesgo cardiovascular.

Métodos: Se efectuó un estudio analítico de cohorte retrospectivo, evaluando las coronariografías realizadas en nuestro centro entre Junio de 2009 a Julio de 2015. Se definió CE como dilatación $>1,5$ veces comparado con el diámetro de la arteria de referencia. Se estudiaron factores de riesgo cardiovascular clásicos y se compararon con un grupo control elegido de forma aleatoria.

Resultados: De 9648 coronariografías, 64 presentaban CE. La mayor parte eran hombres, de menor edad, con menos hipertensión arterial, diabetes e hiperlipidemia, comparados con los controles. En la mayoría de los casos la CE afectaba a 3 vasos $(83,3 \%)$ y en solo 5 casos (28\%) coexistía con estenosis. El análisis multivariado señaló como factores de riesgo significativos a la edad $<55$ años (OR: 2,63, IC: $1,4-$ $4,9, \mathrm{p}<0,05$ ), Obesidad (OR: 3,2; IC:1,7-5,8, p<0,05) e Hiperlipidemia (OR: 0,09; IC: 0,016-0,54). Considerando los pacientes que se presentaron como SCA se observó que los pacientes con CE fueron más jóvenes (45,9 años; DE: 5,9 v/s 48,8 años; DE: 5,3; $\mathrm{p}=0,02)$, y con menos hiperlipidemia (OR:0,2; IC:0,06-0,7, $\mathrm{p}=0,01)$. Respecto a la obesidad, esta fue más prevalente en pacientes con CE (OR: 2,49; IC: 0,956-6,4. $\mathrm{p}=\mathrm{ns})$.

Conclusión: LaCE es una entidad poco frecuente, que puede producir SCA aun en ausencia de estenosis significativa. Son pacientes más jóvenes y con menos antecedente de dislipidemia, por lo que en su patogenia aparentemente participan factores diferentes a los de la enfermedad ateroesclerótica obstructiva.

Correspondencia:

Correspondencia:

Dr. Alejandro Martínez,

amartine@med.puc.cl 


\section{Compared to usual coronary artery disease risk factors are different in those with coronary ectasia}

Background: Coronary ectasia (CE) is an uncommon condition where coronary artery dilatation coexists with atherosclerotic plaques. It may present as either acute or chronic syndromes even in the absence of coronary artery stenosis. Differences in risk factors associated to CE compared to those associated to usual CAD have been described.

Methods: We retrospectively analyzed coronary arteriograms performed between June 2009 and July 2015. CE was defined as the presence of dilatation $>1.5$ times the diameter of the unaffected vessel. Cardiovascular risk factors were compared in $\mathrm{CE}$ vs a random sample of non-CE patients.

Results: Out of 9648 coronary arteriograms 64 showed CE $(9.5 \%)$. Compared to controls, CE patients were males, younger and hat lower prevalence or hypertension, diabetes and hyperlipidemia. CE was present in all 3 main vessels in $83.3 \%$ of CE pa- tients and co-existed with significant stenosis in only $28 \%$. Multivariate analysis showed that significant differences in risk factors were age $<55$ years (OR: 2.63; CI: 1.4 to $4.9, \mathrm{p}<0.05$ ), obesity (OR: 32 ; CI: 1.7 to $5.8, \mathrm{p}<0.05)$ and hyperlipidemia (OR: 0.09 ; CI: 0.016 to 0.54 ). In patients presenting with an acute coronary syndrome, those with $\mathrm{CE}$ were younger ( 45,9 years; SD: $5,9 \mathrm{v} / \mathrm{s} 48,8$ years; SD: 5,$3 ; \mathrm{p}=0,02$ ), $\mathrm{y}$ and a lower prevalence or hyperlipidemia (OR:0,2; IC: $0,06-0,7, \mathrm{p}=0,01$ ).

Conclusion: $\mathrm{CE}$ is an infrequent condition in CAD. It may me associated to either acute or chronic syndromes. They are younger, have a lower prevalence of dyslipidemia suggesting that risk factors other than traditionally recognized in obstructive CAD influence de development of $\mathrm{CE}$

Keywords: coronary artery disease; ectasia; atherosclerosis; aneurysm; coronary angiography. 
Introducción: La coronarioectasia (CE) se define como la dilatación difusa o localizada del lumen coronario mayor a 1,5 veces del diámetro normal de las arterias adyacentes ${ }^{1-6}$. Su prevalencia es variable según las diferentes series, desde 0,3 a $6 \%$ de todos los estudios coronarios ${ }^{2}$, 7-11. Se ha asociado a diversas causas desde enfermedades del colágeno, enfermedades inflamatorias (Kawasaki, Takayasu, poliarteritis nodosa, etc.), origen congénito, pero principalmente se ha asociado a la ateroesclerosis ${ }^{4-5-12}$. Si bien las dilataciones coronarias coexisten con placas ateroescleróticas no está clarificado si corresponden al mismo proceso fisiopatológico, y si así lo fuere, por qué estos pacientes tienen este tipo de remodelado.

Se ha documentado presencia de flujo lento mediante el TIMI frame count en pacientes con $\mathrm{CE}^{13-15}$. El flujo lento que se asocia a la $\mathrm{CE}$, se puede manifestar como angina crónica o síndrome coronario agudo (SCA), independiente de la existencia de estenosis coronaria. La causa podría deberse a microembolia hacia segmentos distales u oclusión trombótica del vaso afectado ${ }^{13-16}$. El tamaño de la dilatación incide en el flujo lento ${ }^{17}$. Así también se ha visto una alteración del flujo microvascular con disminución de la reserva de flujo coronario ${ }^{17-19}$.

Distintas series lo han asociado a perfiles variados de factores de riesgo cardiovasculares. Sus mecanismos patogénicos no se han aclarado, y el remodelado positivo podría corresponder a entidades patológicas diferentes a la enfermedad ateroesclerótica clásica ${ }^{20}$. Si así fuera, es posible que los factores de riesgo asociados a esta patología fuesen diferentes a los de la ateroesclerosis.
El objetivo del estudio fue caracterizar la enfermedad por CE en nuestra población e identificar los factores de riesgo asociados a su presencia.

Métodos: Se planificó un estudio con diseño observacional descriptivo de cohorte retrospectivo. Entre Junio de 2009 a Julio de 2015 se extrajeron de la base de datos todos aquellos pacientes que se sometieron a estudio coronariográfico en el laboratorio de hemodinamia del Hospital Sótero del Río, los que se separaron según presencia o no de CE. Se definió CE como dilatación de arterias coronarias ( $>1,5$ veces de la arteria o segmento de referencia normal). Se analizaron las coronariografías de los pacientes con CE y se estableció la extensión de la enfermedad según la escala de Markis et al ${ }^{21}$ : tipo I, enfermedad difusa con compromiso de 2 o más vasos; Tipo II, enfermedad difusa de un vaso y localizada de otro; Tipo III, Ectasia difusa en 1 vaso; y Tipo IV como ectasia localizada en un vaso (Figuras 1, 2 y 3). Se determinó la presencia de placas ateroescleróticas y si producían estenosis significativa la que se definió como $\geq 50 \%$ en el tronco coronario izquierdo o $\geq 70 \%$ o más en las otras arterias (Figura 2). Se determinó el diámetro máximo del vaso con ectasia y de los segmentos sanos (o de referencia) y se estableció la relación de diámetro de segmento ectásico versus normal (razón ectasia/normal). Para evaluar si existía diferencia entre extensión de enfermedad y variables clínicas, se agrupó a los pacientes según la clasificación de Markis en dos grupos: Grupo 1 (Markis I, II y III) y Grupo 2 (Markis IV) y se analizaron variables clínicas y angiográficas.

Figura 1: Coronarioectasia difusa, Markis Tipo I, A) compromiso de coronaria derecha; B) compromiso de descendente anterior y circumfleja

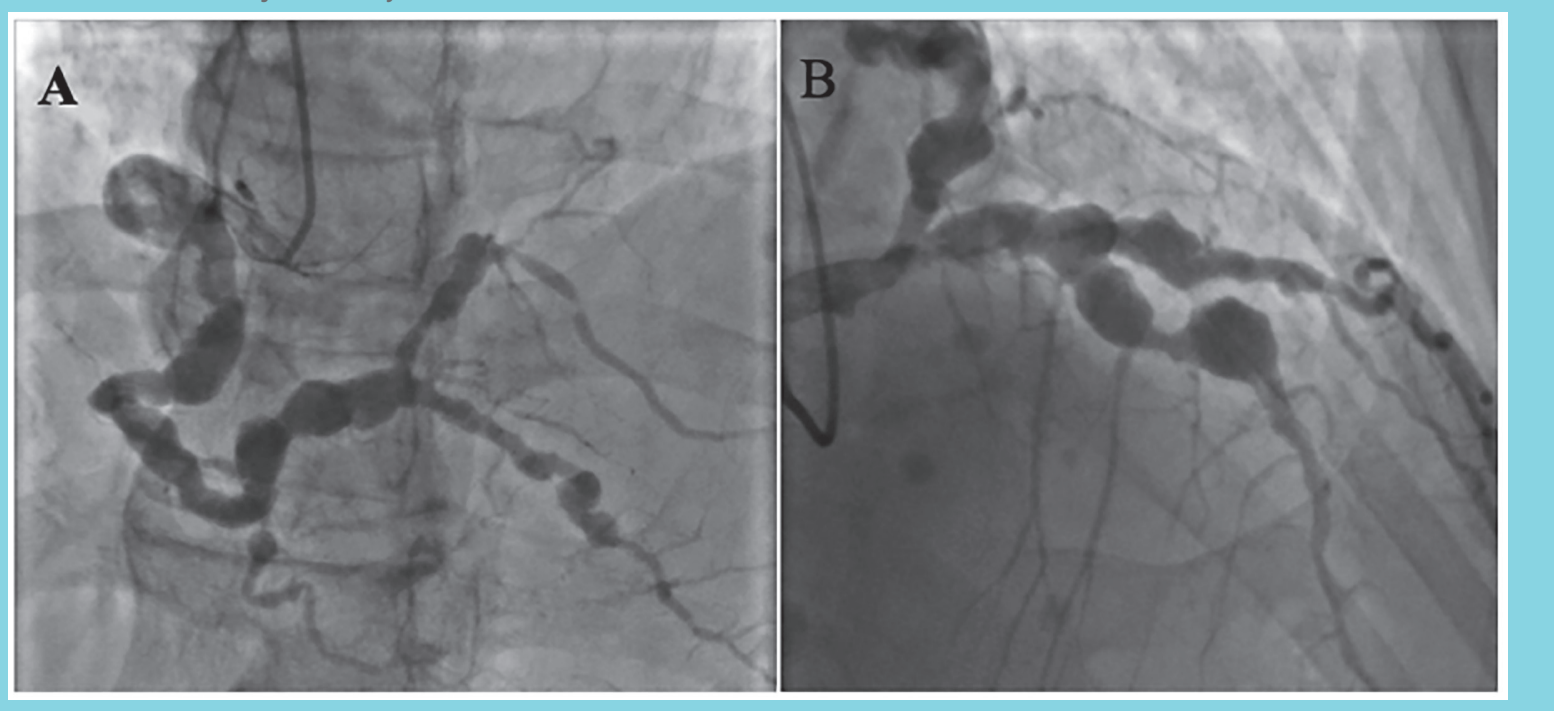


Se comparó a los pacientes que se presentaron como síndromes coronarios agudos, entre los que tenían coronarioectacia con controles elegidos de la base de datos en forma aleatoria. Se excluyeron de este grupo aquellos estudios preoperatorios, estudio pre trasplante, hipertensión pulmonar, valvulopatías y congénitos. Se utilizó una regresión logística univariada y multivariada para estimar los predictores independientes de CE y estos valores se expresan como Odds ratio (OR) con su intervalo de confianza.

Además, se realizó un análisis estratificado por edad (menores de 55 años), excluyendo de este grupo a aquellos con antecedente de cirugía de revascularización, angioplastía, valvulopatía o miocardiopatía preexistente.

Figura 2: Compromiso difuso de circumfleja y descendente anterior (DA) con gran aneurisma en tercio medio de DA posterior a estenosis severa por placa ateroesclerótica (flecha)

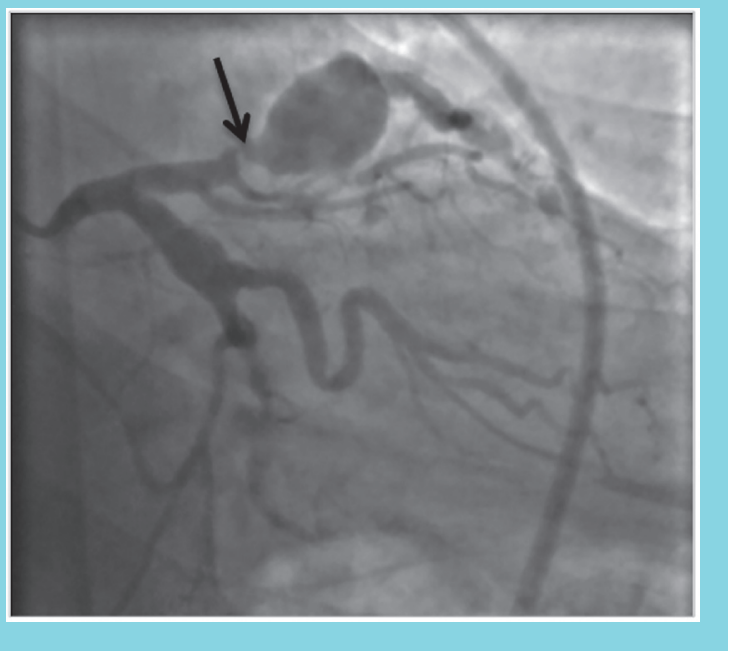

Figura 3: Compromiso localizado coronaria derecha, Markis IV.

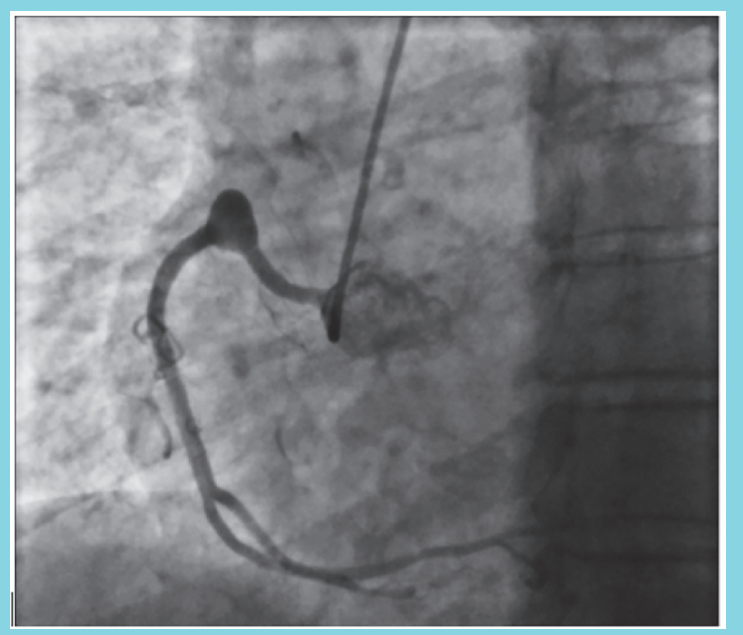

Las variables continuas se expresan como media \pm desviación standard, y las variables categóricas como valor absoluto y porcentaje. La comparación de medias de muestras independientes se realizó mediante t de Student, y la comparación de proporciones mediante test de chi cuadrado. Se consideró significativa una $\mathrm{p}<0,05$. Los datos fueron analizados mediante el paquete SPSS 21.0 para MacOS.

Resultados: Entre Junio de 2009 a Julio de 2015 se realiza un total de 9648 coronariografías, de las cuales 64 presentaban CE. $(0,66 \%)$.

\section{Características clínicas}

Los pacientes con CE fueron principalmente hombres (64\%), más jóvenes que aquellos sin CE $(58,95 \pm 12$ años versus $62,87 \pm 10,5$ años, $p=0,01)$. En el grupo con $\mathrm{CE}$ existió menor prevalencia de factores de riesgo cardiovascular tradicionales como hipertensión arterial $(67,2 \%$ vs $78,4 \%)$, diabetes mellitus tipo 2 ( $31,3 \%$ vs $46,8 \%)$ e hiperlipidemia ( $21,9 \%$ vs $43,6 \%$ ); no existiendo diferencias significativas en tabaquismo, obesidad, uso de cocaína, antecedentes familiares e insuficiencia renal.

Los motivos de indicación del estudio coronariográfico en los pacientes con $\mathrm{CE}$ fueron: síndromes coronarios agudos $(51,6 \%)$, estudio de disnea o insuficiencia cardíaca $(12,6 \%)$, infarto con elevación del segmento ST $(15,6 \%)$, angina crónica o isquemia silente $(10,9 \%)$, evaluación preoperatoria de cirugía cardíaca $(4,7 \%)$ y otros $(4,6 \%)$.

\section{Caracterización angiográfica}

Se vio más frecuentemente afectada la descendente ante-

Tabla 1: Características angiográficas de los pacientes con coronarioectasia.

\begin{tabular}{lr}
\hline ACI & Arterias Coronarias involucradas \\
DA & $5(7,8 \%)$ \\
ACF & $40(62,5 \%)$ \\
CD & $37(57,8 \%)$ \\
& $39(60,9 \%)$ \\
Tipo I & $28(43,7 \%)$ \\
Tipo II & $8(12,5 \%)$ \\
Tipo III & $16(25 \%)$ \\
Tipo IV & $12(18,7 \%)$ \\
& $35(54,7 \%)$ \\
Sin estenosis & $14(21,8 \%)$ \\
Leves & $2(3,1 \%)$ \\
Moderadas & $13(20,3) \%$ \\
Severas & Placas ateroescleróticas \\
\hline
\end{tabular}

$T C I=$ tronco común izquierdo; $D A=$ descendente anterio $; A C F=$ circunfleja; $C D=$ coronaria derecha. 


\begin{tabular}{|c|c|c|c|c|}
\hline & & CE difusa & CE limitada & p \\
\hline & $n$ & 52 & 12 & \\
\hline \multirow[t]{2}{*}{ Sexo } & Femenino & $14(60,8 \%)$ & $9(39,1 \%)$ & \multirow{2}{*}{0,022} \\
\hline & Masculino & $38(92,6 \%)$ & $3(7,3 \%)$ & \\
\hline Edad Media & $61,2 \pm 11,1$ & $56,1 \pm 14,6$ & ns & \\
\hline \multirow[t]{10}{*}{ Factores De Riesgo } & Hipertensión & $34(65,7 \%)$ & $9(75 \%)$ & ns \\
\hline & Diabetes & $15(28,8 \%)$ & $5(41,6 \%)$ & ns \\
\hline & Dislipidemia & $11(21,1 \%)$ & $3(25 \%)$ & ns \\
\hline & Tabaco & $20(38,4 \%)$ & $3(25 \%)$ & ns \\
\hline & Obesidad & $15(28,8 \%)$ & $3(25 \%)$ & ns \\
\hline & Uso de Cocaína & $1(1,9 \%)$ & $0(0 \%)$ & ns \\
\hline & Antecedentes & & & \\
\hline & familiares & $4(7,7 \%)$ & $0(0 \%)$ & ns \\
\hline & Insuficiencia & & & \\
\hline & renal crónica & $3(5,8 \%)$ & $(0 \%)$ & ns \\
\hline \multirow[t]{4}{*}{$\begin{array}{l}\text { Forma de } \\
\text { presentación }\end{array}$} & SCA s/EST & $25(48 \%)$ & $8(66,6 \%)$ & ns \\
\hline & SCA c/EST & $7(13,4 \%)$ & $3(25 \%)$ & ns \\
\hline & Angina Crónica & $6(11,6 \%)$ & $1(8,3 \%)$ & ns \\
\hline & Otros & $13(25 \%)$ & $1(8,3 \%)$ & ns \\
\hline \multicolumn{5}{|l|}{ Arteria } \\
\hline \multirow[t]{3}{*}{ Comprometida } & DA & $35(67,3 \%)$ & $5(41,6 \%)$ & ns \\
\hline & ACF & $35(67,3 \%)$ & $2(16,6 \%)$ & 0,01 \\
\hline & $C D$ & $34(65,3 \%)$ & $5(41,6 \%)$ & ns \\
\hline \multirow[t]{3}{*}{ Tamaño Ectasia } & DA & $5,5 \pm 2,1$ & $3,9 \pm 0,6$ & ns \\
\hline & ACF & $5,1 \pm 1,25$ & $3,5 \pm 0,4$ & ns \\
\hline & $C D$ & $5,5 \pm 1,8$ & $6,1 \pm 2,5$ & ns \\
\hline \multirow[t]{3}{*}{ Razón Ectasia } & DA & $1,74 \pm 0,45$ & $1,43 \pm 0,1$ & ns \\
\hline & ACF & $1,76 \pm 0,37$ & $1,5 \pm 0$ & ns \\
\hline & $C D$ & $1,77 \pm 0,53$ & $2,2 \pm 0,75$ & ns \\
\hline \multirow[t]{4}{*}{ Estenosis Coronaria } & No & $25(48,1 \%)$ & $10(83,3 \%)$ & ns \\
\hline & Leve & $14(26,9) \%$ & $0(0 \%)$ & - \\
\hline & Moderada & $2(3,8 \%)$ & $0(0 \%)$ & - \\
\hline & Severa & $11(21,1 \%)$ & $2(16,6 \%)$ & ns \\
\hline
\end{tabular}

rior $(62,5 \%)$, seguido de la coronaria derecha $(60,9 \%)$ y la arteria circumfleja (57,8\%). La enfermedad comprometió más de 2 vasos en 56\% (Markis Tipo I y II), afectó solamente a un vaso en $44 \%$ (Markis III y IV). Un 54,7\% de los pacientes no tuvieron lesiones coronarias asociadas, $20,3 \%$ presentaron lesiones estenóticas significativas y $3,1 \%$ lesiones moderadas (Tabla 1). La coronaria derecha presentó tendencia a mayor dilatación con un tamaño máximo de ectasia de 5,62 \pm 1,8 $\mathrm{mm}$ versus 5,35 \pm $2 \mathrm{~mm}$ de la descendente anterior y 4,98 $\pm 1,3 \mathrm{~mm}$ de la circumfleja $(\mathrm{p}=\mathrm{ns})$, sin diferencia en la relación de diámetros ectasia/normal entre las diferentes arterias. Del total de pacientes, $18 \%$ tuvo evidencia de trombos al momento del estudio coronario, 62,\% en aquellos con infarto agudo con elevación del ST y $14 \%$ en los síndromes coronarios sin elevación del ST ( $\mathrm{p}=0,007)$.

Variables clínicas y angiográficas según extensión de enfermedad.

Al analizar por grupos de extensión de enfermedad según 


\begin{tabular}{|c|c|c|c|c|}
\hline & & Con CE & Sin CE & $p$ \\
\hline & $n$ & 40 & 1682 & \\
\hline \multirow[t]{2}{*}{ Sexo (\%) } & Femenino & $14(35 \%)$ & $713(42,4 \%)$ & \multirow{2}{*}{ Ns } \\
\hline & Masculino & $26(65 \%)$ & $969(57,6 \%)$ & \\
\hline Edad (años) & Media & $57,55 \pm 12,54$ & $62,87 \pm 10,51$ & 0,01 \\
\hline \multirow{8}{*}{$\begin{array}{l}\text { Factores } \\
\text { De Riesgo }\end{array}$} & Hipertensión & $30(75 \%)$ & 1319 (78,4\%) & Ns \\
\hline & Diabetes & $12(30 \%)$ & 787 (46,8\%) & 0,038 \\
\hline & Dislipidemia & $7(17,5 \%)$ & $734(43,6 \%)$ & 0,001 \\
\hline & Tabaco & $15(37,5 \%)$ & $682(40,5 \%)$ & Ns \\
\hline & Obesidad & $13(32,5 \%)$ & $356(21,2 \%)$ & Ns \\
\hline & Uso de Cocaína & $1(2,5 \%)$ & $11(0,7 \%)$ & ns \\
\hline & $\begin{array}{l}\text { Antecedentes } \\
\text { familiares }\end{array}$ & $3(7,5 \%)$ & $98(5,8 \%)$ & Ns \\
\hline & $\begin{array}{l}\text { Insuficiencia } \\
\text { renal crónica }\end{array}$ & $2(5 \%)$ & $116(6,9 \%)$ & ns \\
\hline
\end{tabular}

la clasificación de Markis en enfermedad difusa (Markis I, II y III) versus enfermedad limitada (Markis IV), se encontró que los pacientes con CE difusa son predominantemente hombres, y no hubo diferencias significativas en las variables clínicas ni formas de presentación. Del punto de vista angiográfico solamente se encontró diferencia en un menor compromiso de la arteria circumfleja cuando existió enfermedad limitada ( $67,3 \%$ versus $16,6 \%, \mathrm{p}=0,01$ ) (Tabla 2).

Según la presencia o ausencia de placas ateroescleróticas, no hubo diferencias en los factores de riesgo. Sin embargo, aquellos sin estenosis coronarias se presentaron en 72,4\% como síndrome coronario sin elevación del segmento ST, en tanto que aquellos con lesiones ateroescleróticas se presentan de esta forma en $31,8 \%$ ( $\mathrm{p}=0,02)$. Además, aquellos sin estenosis coronarias presentaron menos compromiso de la coronaria derecha $(48,3 \%$ versus $77,3 \%, p=0,03$ ) y una menor dilatación de la arteria descendente anterior definido por la razón de ectasia 1,5 $\pm 0,16$ versus $1,9 \pm 0,58(\mathrm{p}=0,04)$.

\section{Predictores clínicos de coronarioectasia}

Se compararon los pacientes con CE con un grupo control de 1682 pacientes, elegidos de forma aleatoria de la base de datos. Las características clínicas de ambos grupos se presentan en la Tabla 3.
Se realizó un análisis univariado y multivariado para definir predictores clínicos de CE. Los factores de riesgo significativamente diferentes fueron la edad $<55$ años (OR: 2,63, IC: 1,4-4,9, p<0,05), obesidad (OR: 3,2; IC:1,7-5,8, $\mathrm{p}<0,05$ ) e hiperlipidemia (OR: 0,09; IC: 0,016-0,54); No existió diferencias significativas para insuficiencia renal crónica, tabaquismo, antecedentes familiares, ni consumo de cocaína.

Al realizar un análisis estratificado por edad< 55 años y presentación como SCA se encontró que los pacientes con CE fueron más jóvenes (45,9 años; DE: 5,9 v/s 48,8 años; $\mathrm{DE} 5,3 ; \mathrm{p}=0,02)$. El único factor asociado fue la hiperlipidemia. Mientras la hiperlipidemia afectaba a 188 pacientes sin CE (47\%), solo la tuvieron 3 pacientes con CE (16,7\%), (OR:0,2; IC:0,06-0,7, p=0,01). Hubo una tendencia a mayor frecuencia de obesidad en los que tenían CE (OR: 2,49; IC: 0,956-6,4. p=ns). De este grupo, en la mayoría de los casos la $\mathrm{CE}$ afectaba a las 3 arterias coronarias $(83,3 \%)$, y solo en 5 casos (28\%) coexistía con estenosis significativa $(>50 \%)$.

\section{Discusión:}

La CE es una enfermedad de rara presentación en nuestro medio con respecto a series publicadas ${ }^{2,7-11}$ y constituye un grupo heterogéneo de condiciones, definidas inicial- 
mente por un parámetro anatómico arbitrario ${ }^{1-6}$.

Desde un punto de vista fisiopatológico pareciera ser que la CE representa una forma particular de remodelado arterial en respuesta a la presencia de aterosclerosis. El fenómeno subyacente a las alteraciones que se generan en la morfología del vaso obedecen a una forma exagerada de remodelado expansivo, producto de la degradación enzimática de la matriz extracelular de la túnica media ${ }^{22}$. Es así como en modelos experimentales se ha observado como la sobreexpresión de metaloproteinasas de matriz se asocia a un remodelado arterial excéntrico ${ }^{5}$, mientras que la supresión de dichas enzimas genera la inhibición de este fenómeno ${ }^{23}$. La acción de las metaloproteinasas produce, además, una grave disrupción de la lámina elástica interna que genera una puerta de entrada desde la íntima a la media para las células inflamatorias que perpetúan y potencian la degradación de la pared ${ }^{5}$. De esta forma la $\mathrm{CE}$ se produce en una pared vascular intensamente inflamada, predisponiendo a inestabilidad de la placa y aumentando el riesgo de eventos cardiovasculares a pesar de mantener un lumen conservado.

Pasa a ser de gran interés, entonces, precisar los factores de riesgo asociados a la $\mathrm{CE}$ que la hacen diferente del proceso ateroesclerótico estenótico habitual. En nuestra serie los pacientes con CE son mayoritariamente hombres jóvenes, fenómeno concordante con las diferentes series ${ }^{2-9-11-24-25}$. También observamos una menor proporción de factores de riesgo como hipertensión, diabetes mellitus tipo 2 y dislipidemia, que se correlaciona con un metanálisis que evidenció que la $\mathrm{CE}$ se asociaba menos a pacientes diabé$\operatorname{ticos}^{2-26}$. Con respecto a la dislipidemia hay datos contrapuestos, donde algunos autores lo asocian a hiperlipidemia familiar y otros no encuentran ninguna diferencia ${ }^{2}$. Estas diferencias clínicas sustentan la hipótesis de que la CE no sólo corresponde a una forma de remodelado diferente de la ateroesclerosis clásica, sino que se comporta más bien como un tipo particular de enfermedad coronaria ${ }^{2-6-27}$.

Esta enfermedad puede manifestarse clínicamente como angina crónica o síndrome coronario agudo (SCA), independiente de la existencia de estenosis coronaria. La causa puede deberse a microembolía hacia segmentos distales u oclusión trombótica del vaso afectado ${ }^{13}, 16$. En nuestra serie la mayoría de los pacientes se presentaron como síndromes coronarios agudos y en aquellos con infarto con elevación del segmento ST la presencia de trombos fue predominante.

Destaca en nuestra serie una mayor proporción de pacientes con afectación de la descendente anterior, en contraste con la literatura que se encuentra un mayor compromiso de la coronaria derecha.

La extensión de la CE según la clasificación de Markis permite definir la cantidad de vasos afectados y qué proporción de aquel vaso se encuentra con dilataciones. Cabe pensar que la afectación difusa (Markis I, II y III), presenta diferencias clínicas y/o patológicas con el compromiso localizado. Sin embargo, en esta serie solamente difirieron estos grupos en que aquellos con enfermedad difusa son predominantemente hombres y con mayor compromiso de la arteria circumfleja.

En la regresión logística la edad <55 años y la obesidad se correlacionaron con la presencia de $\mathrm{CE}$, a diferencia de la hiperlipidemia que fue un factor en contra para presentar CE. En nuestro análisis estratificado por edad y presentación como síndrome coronario agudo la ausencia de dislipidemia fue el único factor asociado a CE. Así también, este subgrupo de pacientes tiene enfermedad difusa con escasa asociación con estenosis significativa. Nuevamente, destaca que la ausencia de trastorno lipídico se asocie a presentar dilataciones aneurismáticas coronarias, ya que en distintas series la presencia de dislipidemia se asocia a esta forma de enfermedad, llevando a pensar a algunos autores que la dislipidemia se asocia a este "remodelado negativo"2. Desconocemos si este hallazgo tiene implicancia fisiopatológica o si existen diferencias en susceptibilidad genética de nuestra población comparada con la literatura. Las diferencias en la afección difusa y con menos asociación de estenosis significativa hace dudar aún si es una forma de remodelado diferente de la ateroesclerosis clásica o una afección distinta ${ }^{3-5-28-29}$.

Este trabajo muestra como las características clínicas, angiográficas y los factores de riesgo de los pacientes con $\mathrm{CE}$ difieren claramente con lo clásicamente conocido de aterosclerosis obstructiva. Se hace necesario entonces, mayor investigación para entender su fisiopatología y caracterizar mejor esta forma particular de enfermedad coronaria.

Deben considerar algunas limitaciones a este trabajo. Al ser seleccionados los pacientes desde la base de datos, existe un sesgo y pérdida de casos que no hayan sido descritos como CE. Así también, sólo estamos viendo a pacientes derivados al laboratorio de hemodinamia, por lo que la real incidencia de esta enfermedad es desconocida y podemos estar evaluando casos más graves o con mayor cantidad de patologías. El bajo número de casos disminuye el poder estadístico de nuestras observaciones. La presencia de factores de riesgo tales como hipertensión, diabetes y dislipidemia fueron recogidos por la entrevista clínica, lo que podría asociarse a un infra diagnóstico por desconocimiento del paciente de esta patología de forma previa. 
En conclusión, la coronarioectasia es una entidad poco frecuente, que puede producir SCA aun en ausencia de estenosis significativa. Son pacientes más jóvenes, con menos dislipidemia y en su patogenia aparentemente participan factores diferentes a los de la enfermedad ateroesclerótica obstructiva.

\section{Referencias}

1. TUNICK PA, SLATER J, KRONZON I AND GLASSMAN E. Discrete atherosclerotic coronary artery aneurysms: a study of 20 patients. J Am Coll Cardiol. 1990;15:279-82.

2. PINAR BERMUDEZ E, LOPEZ PALOP R, LOZANO MARTINEZ-LUENGAS I, CORTES SANCHEZ R, CARRILLO SAEZ P, RODRIGUEZ CARRERAS R, et al. [Coronary ectasia: prevalence, and clinical and angiographic characteristics]. Rev Esp Cardiol. 2003;56:473-9.

3. DENDRAMIS G, PALEOLOGO C, LO PRESTI A, PIRAINO D, LO GRECO V, GRASSEDONIO E, et al. [Coronary artery ectasia: etiopathogenesis, diagnosis and treatment]. G Ital Cardiol (Rome). 2014;15:161-9.

4. MAVROGENI S. Coronary artery ectasia: from diagnosis to treatment. Hellenic J Cardiol. 2010;51:158-63.

5. ANTONIADIS AP, CHATZIZISIS YS AND GIANNOGLOU GD. Pathogenetic mechanisms of coronary ectasia. Int J Cardiol. 2008;130:335-43.

6. SWAYE PS, FISHER LD, LITWIN P, VIGNOLA PA, JUDKINS MP, KEMP HG, et al. Aneurysmal coronary artery disease. Circulation. 1983;67:134-8.

7. HARTNELL GG, PARNELL BM AND PRIDIE RB. Coronary artery ectasia. Its prevalence and clinical significance in 4993 patients. Br Heart J. 1985;54:392-5.

8. ALMANSORI MA, ELSAYED HA. Coronary artery ectasia - A sample from Saudi Arabia. J Saudi Heart Assoc. 2015;27:160-3.

9. AMIRZADEGAN AR, DAVOODI G, SOLEIMANI A, LOTFI TOKALDANY M, HAKKI KAZAZI E, SHABPIRAY H, et al. Association between Traditional Risk Factors and Coronary Artery Ectasia: A Study on 10057 Angiographic Procedures among Iranian Population. J Tehran Heart Cent. 2014;9:27-32.
10. BOLES U, ERIKSSON P, ZHAO Y AND HENEIN MY. Coronary artery ectasia: remains a clinical dilemma. Coron Artery Dis. 2010;21:318-20.

11. VALENTE S, LAZZERI C, GIGLIOLI C, SANI F, ROMANO SM, MARGHERI M, et al. Clinical expression of coronary artery ectasia. J Cardiovasc Med (Hagerstown). 2007;8:815-20.

12. BEFELER B, ARANDA MJ, EMBI A, MULLIN FL, EL-SHERIF N AND LAZZARA R. Coronary artery aneurysms: study of the etiology, clinical course and effect on left ventricular function and prognosis. Am J Med. 1977;62:597-607.

13. PAPADAKIS MC, MANGINAS A, COTILEAS P, DEMOPOULOS V, VOUDRIS V, PAVLIDES G, et al. Documentation of slow coronary flow by the TIMI frame count in patients with coronary ectasia. Am J Cardiol. 2001;88:1030-2.

14. TONY H, MENG K, WU B AND ZENG Q. Among Ectasia Patients with Coexisting Coronary Artery Disease, TIMI Frame Count Correlates with Ectasia Size and Markis Type IV Is the Commonest. Cardiol Res Pract. 2015;2015:282170.

15. KOSAR F, ACIKGOZ N, SAHIN I, TOPAL E, GUNEN H, ERMIS N, et al. Effects of co-existence of coronary stenosis and the extent of coronary ectasia on the TIMI frame count in patients with coronary artery ectasia. Int Heart J. 2005;46:211-8.

16. RAB ST, SMITH DW, ALIMURUNG BN, RAB R AND KING SB, 3rd. Thrombolytic therapy in coronary ectasia and acute myocardial infarction. Am Heart J. 1990;119:955-7.

17. KOSAR F, ACIKGOZ N, SAHIN I, TOPAL E, AKSOY Y AND CEHRELI S. Effect of ectasia size or the ectasia ratio on the thrombosis in myocardial infarction frame count in patients with isolated coronary artery ectasia. Heart Vessels. 2005;20:199-202.

18. AKYUREK O, BERKALP B, SAYIN T, KUMBASAR D, KERVANCIOGLU C and Oral D. Altered coronary flow properties in 
diffuse coronary artery ectasia. Am Heart J. 2003;145:66-72.

19. MANGINAS A AND COKKINOS DV. Coronary artery ectasias: imaging, functional assessment and clinical implications. Eur Heart J. 2006;27:1026-31.

20. SCHOENHAGEN P, ZIADA KM, VINCE DG, NISSEN SE AND TUZCU EM. Arterial remodeling and coronary artery disease: the concept of "dilated" versus "obstructive" coronary atherosclerosis. J Am Coll Cardiol. 2001;38:297-306.

21. MARKIS JE, JOFFE CD, COHN PF, FEEN DJ, HERMAN MV AND GORLIN R. Clinical significance of coronary arterial ectasia. Am J Cardiol. 1976;37:217-22.

22. LI JJ, HE JG, NAN JL, HE ZX, ZHU CG AND LI J. Is systemic inflammation responsible for coronary artery ectasia? Int J Cardiol. 2008;130:e69-70.

23. PRESCOTT MF, SAWYER WK, VON LINDEN-REED J, JEUNE M, CHOU M, CAPLAN SL, et al. Effect of matrix metalloproteinase inhibition on progression of atherosclerosis and aneurysm in LDL receptor-deficient mice overexpressing MMP3, MMP-12, and MMP-13 and on restenosis in rats after balloon injury. Ann N Y Acad Sci. 1999;878:179-90.
24. ZOGRAFOS TA, KOROVESIS S, GIAZITZOGLOU E, KOKLADI M, VENETSANAKOS I, PAXINOS G, et al. Clinical and angiographic characteristics of patients with coronary artery ectasia. Int J Cardiol. 2013;167:1536-41.

25. GUNES Y, BOZTOSUN B, YILDIZ A, METIN ESEN A, SAGLAM M, BULUT M, et al. Clinical profile and outcome of coronary artery ectasia. Heart. 2006;92:1159-60.

26. HUANG QJ, LIU J, CHEN MH AND LI JJ. Relation of diabetes to coronary artery ectasia: A meta-analysis study. Anadolu Kardiyol Derg. 2014;14:322-7.

27. APARICI M, PETEIRO J, FERNANDEZ DE ALMEIDA CA, HIDALGO R, ALZAMORA P, BARBA J, et al. [Coronary ectasis: another form of atherosclerosis]. Med Clin (Barc). 1989;93:368-71.

28. CAY S. Coronary artery ectasia: still an unknown phenomenon. Int J Cardiol. 2010;145:515.

29. SAGLAM M, KARAKAYA O, BARUTCU I, ESEN AM, TURKMEN M, KARGIN R, et al. Identifying cardiovascular risk factors in a patient population with coronary artery ectasia. Angiology. 2007;58:698-703. 